\title{
Proposals for strategic planning to address the current situation of COVID-19 epidemic
}

\section{- Second Communiqué from the College of Community Physicians of Sri Lanka}

\section{The College of Community Physicians of Sri Lanka}

Author group composed of major contributors: Kapila Jayaratne ${ }^{1 *}$, Carukshi Arambepola ${ }^{2}$, Shamini Prathapan $^{3}$; reviewers: Ruwan Ferdinando ${ }^{1}$, Athula Liyanapathirana ${ }^{1}$, Renuka Jayatissa ${ }^{1}$, Sameera Senanayake, Pasyodun Koralage Buddhika Mahesh ${ }^{5}$; Sinhala and Tamil language translators: Enoka Wickremasinhge ${ }^{1}$, Sumudu Avanthi Hewage, Santhushya Fernando ${ }^{1}$, Ishanka Ayeshwari Talagala, Sashiprabha Nawaratne ${ }^{1}$, B Kumarendran ${ }^{6}$; and overall guidance: Sapumal Dhanapala ${ }^{7}$

${ }^{1}$ Ministry of Health and Indigenous Medical Services, Sri Lanka; ${ }^{2}$ University of Colombo, Sri Lanka; ${ }^{3}$ University of Sri Jayewardenepura, Sri Lanka; ${ }^{4}$ Queensland University of Technology, Australia; ${ }^{5}$ University of Melbourne, Australia; ${ }^{6}$ University of Jaffna, Sri Lanka; ${ }^{7}$ World Health Organization

Correspondence: kapjay613@gmail.com

https://orcid.org/0000-0001-9795-4342

DOI: https://doi.org/10.4038/jccpsl.v26i5.8381

Received on 30 March 2020

Accepted on 8 July 2020

The College of Community Physicians of Sri Lanka (CCPSL) endorses the measures taken by the Government of Sri Lanka including health authorities to adopt a "whole-of-government, whole-of-society" approach centred on science-based and law-based strategies for controlling the COVID-19 epidemic in compliance with the World Health Organization (WHO) recommendations.

The CCPSL places on record the active and substantial contribution of the entire public health hierarchy from the grass root level public health inspectors and public health midwives to the highest level public health specialists, policy makers and administrators in various capacities and facets of the epidemic response. Except a few sporadic incidents of social irresponsibility reported, the response of the general public to adopt social distancing has also been satisfactory. This momentum needs to be continued, if we are to achieve the maximum through the intense public health response.

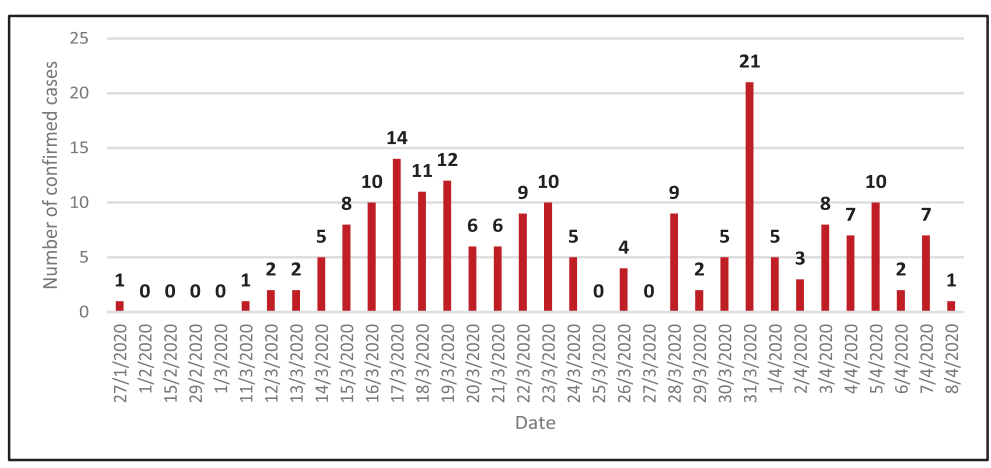

Figure 1: Number of confirmed COVID-19 cases in Sri Lanka 
As of 8 April 2020, in Sri Lanka, there were 186 confirmed cases with 7 deaths (Figure 1). We are now in stage 3 of the epidemic, with clustering of the cases within families or in villages. Every effort has been made to prevent progression of the disease to the next stage, i.e. community transmission, since it would be very difficult to stop the increase of cases if we move into stage 4 . The country's position in relation to some other Asian countries is shown in Figure 2.

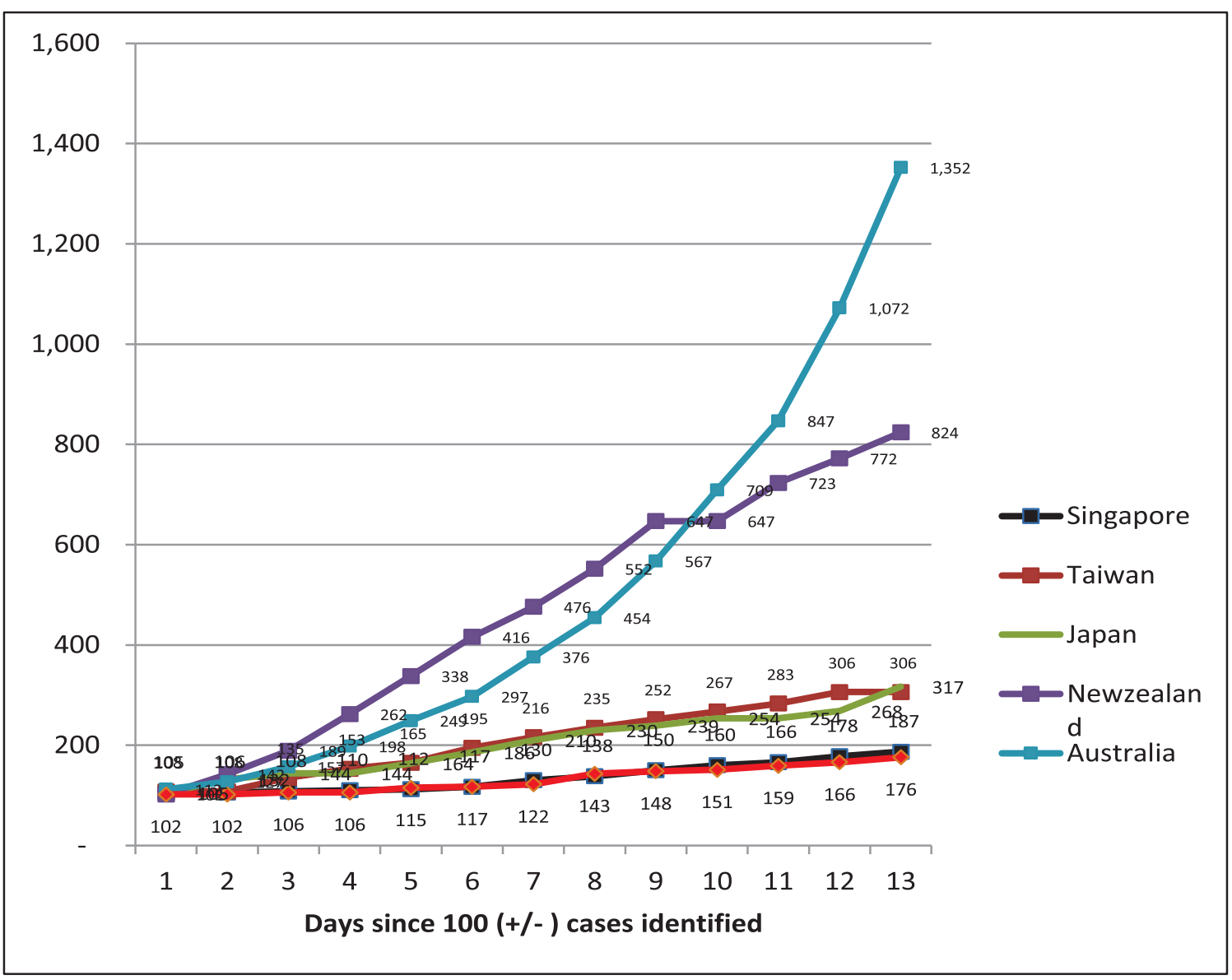

Figure 2: Accumulated number of confirmed COVID-19 cases of selected Asian countries

It is apparent that the country has been and will be successful in flattening the curve. However, the better position of Sri Lanka should be interpreted and weighed against the specific country contexts and proportion of testing conducted per million population. This prompts us to continuously monitor the status of the epidemic and to regularly review and update the strategies implemented for their effectiveness.

As the apex professional body in the practice of public health in Sri Lanka, the CCPSL has agreed upon the following proposals to be considered in the strategic planning of the epidemic.
1. Streamline the national and sub-national coordination of the epidemic response

Effective coordination at both ground and national levels is the key to success in the epidemic response. There will be multiple challenges when different specialties and various non-health sectors are involved in the process. We see several issues both at national and sub-national levels when implementing the prescribed protocols. It is apparent that some of the higher-level stakeholders are not fully involved in this process and multiple instances of noncompliance with guidelines at ground level. An immediate revisit is needed to sustain the effective 
coordination from top to bottom, between the curative and public health sectors as well as between relevant divisions within each sector.

\section{Employ the WHO strategy "Test, Test, Test"}

WHO advocates "If countries detect, test, treat, isolate, trace, and mobilize their people in the response, those with a handful of COVID-19 cases can prevent those cases becoming clusters, and those clusters becoming community transmission". One of the main objectives of COVID-19 testing is to find and isolate asymptomatic carriers, before they spread the infection unwittingly. Evidence from South Korea and Singapore implies that flattening of the epidemic curve is achievable with aggressive testing strategy. In the Sri Lankan context, increased detection of cases and carriers reduces the need for more stringent government measures, like curfew and movement restrictions.

During the initial response, Sri Lanka adopted fairly strict criteria on who should be tested for COVID-19. As the epidemic evolves, this slow rollout of testing and limited testing capacity may blunt the response to the epidemic. We observe that reasons for not carrying out more testing would be due to multiple logistics problems. Currently, reverse transcription polymerase chain reaction (RT-PCR) testing is done only at 7 centres (Medical Research Institute (MRI), Kandy Teaching Hospital (TH), Anuradhapura TH, Karapitiya TH, Ragama TH, Infectious Disease Hospital and Sri Jayewardenepura University), while there are many laboratories all over the country which have GeneXpert machines, which could perform the testing, provided the FDA approved kits are available for this purpose.

We propose the following:

a. Revise the testing criteria expanding to cover more suspected cases and contacts with clear prioritization strategy by a technical team

b. Employ volunteering medical laboratory technicians (MLTs) in other government laboratories and scientists with real time PCR experience at laboratories with unutilized GeneXpert machines c. Establish a larger central laboratory (at MRI) with some of the unutilized GeneXpert machines and employ volunteering MLT/Scientists

d. Adopt pooling of samples for PCR, which can exponentially increase the testing capacity

e. Explore the rationale and feasibility of rapid $\mathrm{Ag}$ or Ab based serological testing

In keeping with the high testing rates expected following the recently revised screening criteria, a prioritizing mechanism should be worked out for the target groups being tested. Further, other options should be considered to prevent hospitals getting overloaded with test positives. Self-isolation/separate centres should be considered for asymptomatic or mild cases.

\section{Scientific prediction of the epidemic}

A pre-requisite of the epidemic response to COVID19 is the availability of a near valid country-specific model to predict the progression of the epidemic. This would address the burning issue of the general public- when and how will this epidemic end? As key stakeholders, there is a responsibility towards providing a sound prediction on the course of the epidemic, based on a country-specific model generated using local data. In this regard, the CCPSL recommends that this task be entrusted to a panel of experts representing different fields, and the model tested with actual data. Once available, the CCPSL opines that it should be further refined by the relevant professional groups and utilized during planning of the epidemic response and to plan out the post pandemic response.

At present, there is no ready access to much needed COVID-19 data for meaningful utilization. It would be counter-productive to release data once the epidemic is over. It is important to initiate a thorough epidemiological investigation into the disease in order to understand the local picture. The CCPSL strongly believes that data collected at national level be utilized for action- to understand many aspects of the local disease, such as infectivity, clinical presentation and course, testing outcomes, which are not yet fully known. These interpretations including 
the $\mathrm{R} 0$ and Rt computed for different phases will give direction to revise the case definitions, testing criteria and to plan ahead. It should be emphasized that comprehensive individual data should be made available for technical experts for analysis, forecasting and formulating preventive strategies. As a college with collective resources, the CCPSL is able to officially provide our expertise for on-demand evidence compilations and data analysis to assist the Task Force to address the local situation, controversial issues and provide evidence-based direction.

\section{Scenario-based approach}

The CCPSL observes that a crisis management strategy would not be successful in the long run. In response, it is necessary to go for a scenario-based modality to further control the epidemic, which could well be guided by a valid prediction model. Potential country scenarios should be worked out and strategies should be formulated beforehand. Following are some of the scenarios we think that the country would encounter.

Scenario -1 : Community transmission and exponential increase of cases with preventive measures going out of control (This would be the worst scenario)

Scenario - 2: Current scenario with sporadic cases and no burden to the health system

Scenario - 3: Lowered health system capacity due to infected healthcare workers or reduced supplies

Scenario-4: Second wave of epidemic

Building different predictable scenarios and suggesting appropriate actions should be delegated to a team of professionals with public health expertise.

\section{Clear guidance on wearing masks}

It is now known that the virus can spread between people interacting in close proximity, even if those people are asymptomatic / pre-symptomatic. Some countries have shown positive results achieved through facemasks, however its application across the board as a preventive strategy for the public is debated, in view of many logistic issues including shortage of facemasks. The CCPSL is of the view that it should be emphasized as an additional voluntary public health measure. Further, the relevant professional colleges and institutes should collectively review the evidence available and guidelines provided on this, in order to make recommendations that are favourable in the local context. This review should specifically consider the evidence available on non-surgical masks and identify the instances where its use could be of health benefit, so that more customized health messages could be generated for the general public, rather than having a rigid approach on its use.

\section{Self-quarantine vs quarantine at centres}

In some situations, self-quarantine poses major challenges on compliance and ultimately leading to negative outcomes. In such situations, quarantine at centres should be considered for individuals who would be judged to be unreliable by the medical officer of health/ public health inspector. Also, if the exposed person is in a small housing unit without adequate space for home quarantine, then institutional quarantine for the family should be done. The CCPSL believes that rights for the good of the whole population should not be compensated for the loss of personal liberties. Well-coordinated efforts of the public health experts from both health department and armed forces should be strengthened for improving the process at the designated quarantine centres.

\section{Care for the COVID-19 patients}

With regards to care provided for COVID-19 patients, the highest-level decision making is essential in their clinical management. Care of specific population groups (e.g. maternal and new-born care, elderly care) should be given prominence. They should be managed by experienced clinicians, as a multi disciplinary team, with necessary equipment and other logistics be readily available. It is also important to learn from regular reviews of the patients recovered and deaths reported so far, for which case scenarios should be developed and reviewed in a no-fault finding modality to translate lessons learnt into practice, programmes and policy. 


\section{Care for the non-COVID-19 patients}

It is equally important for the country to prevent both direct mortality from the outbreak and indirect mortality from other treatable conditions. WHO reminds governments, hospitals to continue to provide essential health services despite COVID-19 crisis. The country's annual outpatient and inward patient turn out are approximately 7 million $(19,200$ per day) and 56 million (153,400 per day) respectively. In many hospitals, including tertiary care institutes, the epidemic response has created a situation where all medical services are prioritized for COVID-19 patients, to the extent of other patients being denied access and availability to services. On one hand, the people fear to come for services and present late as emergencies, and on the other, when patients do come, the health staff responsiveness would be negative, with a tendency for refusal/postponement care which can result in increased mortality and morbidity that could have been prevented.

Essential care is being misinterpreted also as emergency care due to routine services being disrupted. At present, the entire health system is geared to face the challenge of the epidemic at the expense of services at first contact level, clinic-based care, field health services (child well baby/ immunization / antenatal /family planning), surgical care and other routine medical interventions. We believe the disruption of the whole spectrum of the routine services in the entire country is unwarranted. The consequences of this strategy would end in a long-term catastrophe. As a country with a relatively low COVID-19 caseload, it is essential to maintain the routine service delivery, until strategic shifts are required to ensure maximum benefit for a population, as a result of increasing caseloads or infected health workers.

Health administration should facilitate all the tertiary care or main hospitals in each district to function in full force with necessary precautions, while fever patients should be admitted to identified hospitals for further testing and care. Further, maintaining supply chains for medications and other needed supplies should be done by a high-powered team, as importation may be compromised due to high demand and limited supplies from manufacturing countries.

\section{Exit strategy of the modified 'lockdown'}

The modified "lockdown" strategy adopted by the country is only to flatten the curve and to gain time for the health system to respond. This alone will not work and simultaneous case detection, contact tracing and isolation/quarantine should be employed. The purpose of this strategy is to reduce reproduction of the virus (i.e. Ro, the number of secondary cases which one case would produce in a completely susceptible population). The target is to keep Ro $<1$, which indicates each case infecting less than one person on average. It is essential to review whether the country has achieved this goal. The CCPSL is happy to be involved in such a review and provide expertise.

Ending the curfew too soon could lead to a second outbreak, while enforcing it for too long could further cripple the economy and public morale. It should not lead to a human tragedy as in other countries; thus, a calculated decision making should be undertaken considering all facets of the epidemic and the basic demands of the general public.

Mapping the districts where cases and contacts are most and relaxing curfew in the rest of the districts; and continuing the ban on inter-district migration are some of the strategies that could be adopted for a more targeted response. Further, the essential services such as healthcare centres, supermarkets, groceries, banks and pharmacies should be kept open daily and people allowed to walk to these places ensuring physical distancing. The local industries should be facilitated to open early, while all transport should only allow $50 \%$ of the seating capacity to ensure physical distancing.

\section{Disease stigma}

The stigmatization continues to be a major hindrance for case detection and contact tracing despite new guidelines issued by Director General of Health Services of the Ministry of Health (DGHS) to the media. The efforts should originate from healthcare 
staff up to the general public. Revelation of certain personal identifiers by different health officials to the media should be immediately stopped. Stigmatizing of the health workers and workers in the high-risk districts should be countered.

Strict rules should prevent media coverage of negative community behaviour such as overcrowding. Instead, positive actions such as social distancing should be portrayed and glorified. There should not be a media coverage on the cremation of dead COVID-19 patients. Public should also be educated not to ostracize patients in self-quarantine.

\section{Updating general public}

The CCPSL requests for clear-cut regulations for media spokespersons, to prevent the public getting confused over mixed messages especially on areas where the public has no active role to play. This will prevent the media being used as a platform for conveying personal opinions. There should be strict rules to prevent media from portraying nonauthorised persons giving messages to the public without sanction from the National Task Force. In this regard, it is requested for one spoke person to be officially responsible for giving updates on the lineup.

\section{Learn from success/failures of other countries}

The mainstay of Korea's success was mass indiscriminate testing followed by rigorous contact tracing and the quarantine of close contacts. In China, it was through strict law-based and science-based strategies. The CCPSL suggests that these lessons be taken, analysed, and applied pragmatically suitable for the local context.

We, the public health community, have returned substantial profits to the nation for investing in public health. Sri Lanka can set an example to the entire world, with its timely and effective response to the global pandemic despite being a low- and middleincome country. We take this opportunity to appreciate the political leadership, all the governmental hierarchy, dedicated healthcare staff and most importantly the general public for their contribution in controlling the epidemic.

College of Community Physicians of Sri Lanka

9 April 2020 